\title{
Understanding the interplay between size, morphology and energy gap in photoactive $\mathrm{TiO}_{2}$ nanoparticles $\uparrow$
}

\author{
Ángel Morales-García ${ }^{\mathrm{a}}$, Antoni Macià Escatllar ${ }^{\mathrm{a}}$, Francesc Illas $^{\mathrm{a}}$, Stefan T. Bromley ${ }^{\mathrm{a}, \mathrm{b}^{*}}$
}

a'Departament de Ciència de Materials i Química Física \& Institut de Química Teòrica i Computacional (IQTCUB), Universitat de Barcelona, c/ Martí i Franquès 1-11, 08028 Barcelona, Spain

bInstitució Catalana de Recerca i Estudis Avançats (ICREA), Passeig Lluis Companys 23, 08010

Barcelona, Spain

E-mail: s.bromley@ub.edu

\begin{abstract}
$\mathrm{TiO}_{2}$ nanoparticles (NPs) exhibiting the anatase crystal structure can photocatalyse water splitting using the UV fraction of sunlight, to provide hydrogen fuel in a clean and sustainable manner. However, such NPs have optical energy gaps that cover a small range of relatively high energy solar photons, and thus their photo-efficiency is rather low. Experimentally, NP morphology and size can often be tailored to strongly affect their electronic structure and, consequently, their photocatalytic efficiency. Although anatase NPs with diameters between 10-20 $\mathrm{nm}$ thermodynamically prefer crystalline faceted morphologies, application of various physico-chemical procedures can give rise to more rounded NPs with amorphous shells. Such engineered metastable core-shell NPs, generically referred to as black $\mathrm{TiO}_{2}$, have relatively reduced band gaps due to shell-induced band edge broadening, and thus higher

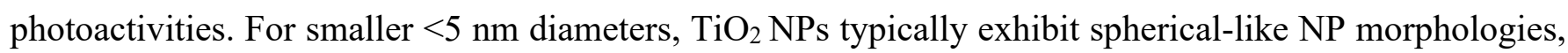
which also display enhanced photoactivity. For such small NPs it is extremely difficult to accurately determine their thermodynamic stability and internal atomic structure experimentally, in order to help rationalise their higher photoactivity. By means of accurate electronic structure calculations we establish the relative stability of spherical and faceted stoichiometric $\mathrm{TiO}_{2} \mathrm{NPs}$ as a function of size with diameters ranging between 1-3.4 $\mathrm{nm}$. Mirroring experimental preparation methods, we also take into account thermal annealing in our NP models. Thermal annealing is found to significantly stabilise relaxed spherical NPs cut from the anatase crystal. In particular, we find that the smallest spherical NPs considered become amorphized by annealing, but, for diameters $>2 \mathrm{~nm}$, annealing can lead to NPs with anatase-cores and amorphous-shells. Much like larger black $\mathrm{TiO}_{2}$ core-shell NPs, we confirm that our core-shell NPs are metastable with respect to faceted anatase NPs. These annealed core-shell NPs also
\end{abstract}


have significantly smaller calculated optical gaps than faceted NPs, which, moreover, match very well with available experimental data, strongly supporting the validity of our core-shell NP structures. Analysing the energy gap narrowing in these core-shell NPs reveals a broadening of the valence band states induced by the amorphous shell, analogous to the general mechanism proposed for black $\mathrm{TiO}_{2} \mathrm{NPs}$. As our NPs are stoichiometric, our results also show that such a band narrowing effect does not intrinsically require the disordered shells to be non-stoichiometric or for incorporation of other atom types. Instead, we show that this effect can simply arise from the reduced coordination of atoms in the amorphous shell. Our careful and systematic computational investigation, using NP models of unprecedented realism, thus provides direct confirmation that the enhanced photoactivity in small spherical $\mathrm{TiO}_{2}$ NP observed in experiment is due to the formation of metastable core-shell NPs. In general, our study provides new detailed insights into the interplay between size, stability, morphology and photoactivity for nano- $\mathrm{TiO}_{2}$.

$\dagger$ Electronic supplementary information (ESI) available. Parameters for the fits reported in Fig. 1 are compiled in Table $\mathrm{S} 1$. In addition, the $\mathrm{O}_{\text {gap }}$ values calculated using PBE functional are included in Fig. S1 together with those estimated by using the PBEx hybrid functional. Finally, the atomic coordinates of all faceted and annealed spherical $\mathrm{TiO}_{2}$ NPs are compiled in the xyz format. See DOI: .... 


\section{INTRODUCTION}

Titania $\left(\mathrm{TiO}_{2}\right)$ is a multifunctional semiconducting material that is used in many practical applications due to its excellent stability, remarkable physical and chemical properties and low cost. ${ }^{1,2}$ The discovery that $\mathrm{TiO}_{2}$ can split water under ultraviolet (UV) light, ${ }^{3}$ in particular, has led to ongoing intensive research in areas such as solar fuels, photovoltaics, and environmental remediation. ${ }^{4-9}$ Bulk titania polymorphs exhibit band gaps of $\geq 3 \mathrm{eV},{ }^{10}$ enabling them to capture only $\sim 10 \%$ of the photons from the sunlight reaching the Earth surface, thus limiting their practical use. Incorporation of noble metals, formation of heterojunctions with other semiconductors, use of dopant agents, and the generation of intrinsic defects such as oxygen vacancies have been studied as potential means to improve the photoactivity and solar use of $\mathrm{TiO}_{2}{ }^{11-15}$ The photocatalytic activity of $\mathrm{TiO}_{2}$ is also a fundamental nanoscale phenomenon, which emerges in suitably sized nanoparticles (NPs). Under ambient conditions, bulk $\mathrm{TiO}_{2}$ thermodynamically favours the rutile crystal structure, however, the photoactive anatase polymorph is mainly observed at the nanoscale due to its lower surface energy. ${ }^{16}$ The performance of anatase $\mathrm{TiO}_{2}$ in many applications largely depends on its optical, electronic, structural, morphological, and surface properties, which can all be tuned through the use of NPs with different sizes, morphologies, crystallinities, and surface facets. ${ }^{4,17-}$ 19 Of particular note in this respect are so-called black $\mathrm{TiO}_{2}$ NPs which are morphologically and structurally manipulated to absorb light in the visible energy range, thus covering a greater range of solarradiation with concomitant efficiency in photocatalysis applications. ${ }^{20}$ Clearly, the design of tailored anatase $\mathrm{TiO}_{2}$-based nanotechnologies relies on both the ability to synthesize appropriate NPs with wellcontrolled sizes and morphologies, and a detailed understanding of how the intrinsic physical and chemical properties depend on these two factors. To enhance the efficiency of NP-based photocatalysts, it is usually desirable to decrease the NP size to be as small as possible to increase the proportion of available surface area while maintaining the intrinsic activity of the material. Such considerations, however, should also take into account the fact that decreasing the size of an anatase NP will also increase quantum confinement which will act to increase the optical energy gap, and thus reduce photoefficiency. ${ }^{21}$ Both experiment ${ }^{22}$ and theory ${ }^{23,24}$ have pointed to a lower size limit of $2-3 \mathrm{~nm}$ for the anatase phase, beyond which the NPs are too small to maintain any crystallinity. Our detailed theoretical study focusses on relative stabilities and properties of realistic NPs with different morphologies, structures, and crystallinities in this important nanosized regime in order to rationalise experimental results and to provide insights into how photoactivity can be enhanced in these systems.

Several synthetic strategies have demonstrated that the morphology of $\mathrm{TiO}_{2}$ NPs depends significantly on the Ti precursor and the surfactants used. ${ }^{25-28}$ Nonhydrolytic and surfactant-assisted solvothermal methods appear to be most effective for both controlling NP size and shape and producing NPs with high crystallinity. ${ }^{29}$ Anatase $\mathrm{TiO}_{2}$ NPs with tailored morphologies in the $10-100 \mathrm{~nm}$ size range have been successfully synthesised by using approaches based on a seeded growth technique. ${ }^{30}$ Both bipyramidal and truncated octahedral anatase $\mathrm{TiO}_{2}$ NPs have been reported. ${ }^{30}$ The bipyramidal NPs 
exhibit (101) surfaces only whereas the truncated octahedrons exhibit (001) and (101) surfaces. Fazio et al. have argued that partial dissolution of these faceted $\mathrm{TiO}_{2} \mathrm{NPs}$ could lead to the formation of hydroxylated NPs with diameters $(\varnothing)$ between $3-5 \mathrm{~nm}$ that exhibit more curved surfaces. ${ }^{31}$ Further theoretical work has examined the structural and electronic properties of partially hydroxylated anatase $\mathrm{TiO}_{2}$ NPs with faceted and spherical morphologies and diameters of $\sim 3 \mathrm{~nm}{ }^{18,32,33}$ These studies suggest that the interplay between hydroxylation and morphology is significant for enhancing the hole trapping ability in spherical NPs, while being less important for faceted NPs. ${ }^{33,34}$ All these theoretical works employ cuts from the anatase crystal structure to derive their spherical and faceted NP models. Removal of low coordinated $\mathrm{Ti}$ atoms and subsequent hydroxylation of remaining relatively low coordinated centres on the surfaces of these cuts are then used to produce stoichiometric hydroxylated NP models, which are then structurally relaxed. This well-defined protocol has the drawback that it produces NPs with uncontrolled varying degrees of hydroxylation dependent on the size and morphology of the original crystal cut. Use of such models, thus makes it difficult to assess the intrinsic relative stabilities of different NPs families with respect to size, morphology and internal structure. Calculations applying thermal annealing to hydroxylated spherical anatase NPs have also resulted in different predictions as to what extent this treatment has on the NP electronic structure. ${ }^{18,35}$ Where some electronic change was calculated, ${ }^{35}$ the intrinsic reason for the effect was not clearly ascribed.

Herein, we focus on a varied set of stoichiometric anhydrous NPs with a range of sizes, for which a more controlled comparative analysis of stability, structure and properties can always be made. Bare anhydrous spherical anatase $\mathrm{TiO}_{2}$ NPs have also been produced in numerous experiments. ${ }^{36,37}$ This indicates that, although hydration may sometimes play a role in promoting more spherical NPs, it is not necessary to maintain the stability of such NPs. As such this raises fundamental questions about the intrinsic stability and electronic/optical properties of anhydrous $\mathrm{TiO}_{2} \mathrm{NPs}$ with respect to their morphology and size.

For anhydrous stoichiometric $\mathrm{TiO}_{2}$ NPs with $\sim 1 \mathrm{~nm}$ diameters, global optimisation searches have established that non-crystalline NPs with quasi-spherical morphologies are the most energetically stable. ${ }^{23}$ For larger sizes, the atomic and electronic structure of anhydrous faceted anatase $\mathrm{TiO}_{2} \mathrm{NPs}$ with maximum diameters up to $6 \mathrm{~nm}$ have also been recently theoretically investigated. ${ }^{38}$ Towards the upper end of this size regime, truncated octahedral and bipyramidal NP structures are found to be almost energetically degenerate with one another, with both exhibiting a low degree of structural distortion relative to the bulk anatase crystal. ${ }^{39,40}$ By interpolating between these two size regimes, it has been estimated that faceted crystalline NPs become more stable than non-crystalline quasi-spherical NPs for a diameter range of $2-3 \mathrm{~nm} .{ }^{23}$ This estimate is in line with studies of experimentally prepared anhydrous spherical NPs, where crystallinity is found to be severely diminished in $\sim 2 \mathrm{~nm}$ NPs due to the structurally disruptive influence of the surface atoms. ${ }^{22}$ This result is also consistent with atomistic simulations of stress in $\mathrm{TiO}_{2}$ NPs. ${ }^{24}$ As kinetic factors can strongly influence the morphology of small NPs in 
experiment, the thermodynamically preferred morphologies of $\mathrm{TiO}_{2} \mathrm{NPs}$ in the $\leq 5 \mathrm{~nm}$ diameter range has not yet been established. Computational modelling offers an alternative, controlled approach to assess whether observed NP morphologies are determined mainly by intrinsic stability (e.g. crystalline or noncrystalline structure), preparatory conditions (e.g. kinetic trapping), or by the NP's environment (e.g. hydration). Herein, we use state-of-the-art electronic structure methods and nanoscale models of unprecedented realism to provide a predictive guide to the stabilities and properties of $0.5-3.5 \mathrm{~nm}$ diameter stoichiometric $\mathrm{TiO}_{2}$ NPs and how they depend on morphology, size and crystallinity. Specifically, we pay particular attention to the accurate modelling of anhydrous NPs with spherical morphologies and, as in experiment, ${ }^{41,42}$ we apply thermal annealing to all our NPs. We compare the stabilities of such annealed NPs both with NPs obtained from simple relaxed spherical cuts and with faceted NPs, enabling us to provide a tentative stability versus size diagram for the $\mathrm{TiO}_{2} \mathrm{NP}$ size range considered. Moreover, we estimate the lowest optical transition by inspecting the energy gaps of all our NPs and show that our annealed stoichiometric spherical NPs have reduced gaps which match particularly well with those from experiments on small spherical NPs with enhanced photoactivity. Analysis of the underlying reason for the gap reduction in spherical NPs points confirms the existence of gap edge broadening due to their core-shell structure, fully in line with the analogous effect in larger black $\mathrm{TiO}_{2}$ NPs. ${ }^{20,43}$ Specifically, we are able to ascribe the gap narrowing to atoms with reduced coordination environments in the surface amorphized shells of our stoichiometric NPs.

\section{RESULTS AND DISCUSSION}

We consider four sets of stoichiometric $\mathrm{TiO}_{2}$ NPs: $(i)$ bipyramidal and cuboctahedral faceted NPs, (ii) spherical NPs directly cut from the anatase crystal structure, (iii) spherical NPs from (ii) which have been thermally annealed, and (iv) quasi-spherical globally optimised NPs. We consider $\left(\mathrm{TiO}_{2}\right)_{n} \mathrm{NPs}$ with $n$ values ranging between 29 - $595 \mathrm{TiO}_{2}$ units, with the largest NP containing 1785 atoms, see Fig. 1 (atomic coordinates of all NPs are provided in the ESI $\dagger$ ). To assign a common measure of diameter for each of these four sets of $\left(\mathrm{TiO}_{2}\right)_{n} \mathrm{NPs}$, we use the diameter of sphere containing $n \mathrm{TiO}_{2}$ units, with the volume of a single $\mathrm{TiO}_{2}$ unit taken as that in bulk anatase. A similar approach was used in recent study comparing morphologically diverse $\mathrm{ZnO} N \mathrm{NP}^{44}$ As noted above, it has been estimated that the anatase structure can persist in NPs down to a $\sim 2-3 \mathrm{~nm}$ size, 22-24 which strongly suggests that this is a nanoscale region where NPs of different morphologies and crystallinities could be energetically competing. Our $\mathrm{TiO}_{2}$ NP models have sizes throughout the range $\sim 1.0-3.4 \mathrm{~nm}$ and thus cover this interesting size regime. In order to obtain accurate structures and energies all NPs are structurally relaxed employing all-electron density functional theory (DFT) based calculations carried out using the PBE exchange correlation functional $^{45}$ as implemented in the FHI-aims code ${ }^{46}$ (see further details in the Computational Methods section). In Fig. 2 we show the size-dependent energetic stabilities of faceted (blue), spherical (red), annealed spherical (green), and global optimized ${ }^{23}$ (pink) $\mathrm{TiO}_{2} \mathrm{NPs}$. The later set contains $\left(\mathrm{TiO}_{2}\right)_{n}$ 
nanoclusters with $\mathrm{n}=28,35$ and 38 (see Fig. 1). The relative stability of all $\mathrm{TiO}_{2} \mathrm{NPs}$ in Fig. 2 is given per $\mathrm{TiO}_{2}$ unit with respect to that of bulk the anatase phase. From the so-called spherical cluster approximation (SCA), ${ }^{47}$ it is assumed that the energetic stability of a $\left(\mathrm{TiO}_{2}\right)_{n} \mathrm{NP}, E_{n}$, relative to that of bulk anatase, $E_{b u l k}$, can be approximately fitted by a scaling law following

$$
E_{n}=E_{b u l k}+a_{1} n^{-\alpha}
$$

where $a_{1}$ is a fitting parameter related the NP morphology, and $\alpha$ is taken to be $1 / 3$ reflecting the surface area to bulk ratio and both $E_{n}$ and $E_{\text {bulk }}$ are per $\mathrm{TiO}_{2}$ unit. Note that equation 1 is derived based on consideration of the geometric shape of a NP only, whereas realistic NPs are more complex entities composed of interacting aggregates of atoms/ions. As such, other contributions to $E_{n}$ (e.g. surface stresses) can become significant which can be approximately included through the inclusion of higher order terms $\left(\right.$ e.g. $\left.a_{2}\left(n^{-1 / 3}\right)^{2}, a_{3}\left(n^{-1 / 3}\right)^{3}, \ldots\right) .{ }^{16,23,44}$ Further details and fitting parameters employed to construct the plot in Fig. 2 are provided in the SI.

From the fitted curves in Fig. 2, we first note that the crossover from non-crystalline NPs (pink) to crystalline facetted NPs (blue) occurs at $n=280$. In a previous study,23 this crossover was estimated to occur at a smaller value of $n=125$. Firstly, we point out that the diameter of an NP with $n=125(\varnothing=$ $2.60)$ and $n=280 \mathrm{TiO}_{2}(\varnothing=2.68 \mathrm{~nm})$ is very similar, and thus the original prediction that this crossover occurs for NP sizes between $2-3 \mathrm{~nm} 23$ is confirmed. The relatively small upward shift of the specific size for the crossing point of the two fitted lines is mainly due to the inclusion of extra data points for large faceted NPs in the present work, which slightly improves the fit for this family of NPs.

In Fig. 2 we also can compare the relative stability of faceted (blue) versus spherical NPs (red and green) as a function of NP size. For spherical bulk-cut NPs that are relaxed to their nearest local minimum energy structure following a standard energy minimization (red), we find that relaxed faceted $\mathrm{TiO}_{2}$ NPs (blue) are more energetically stable for all NP sizes. For NPs with $\varnothing \geq 2 \mathrm{~nm}$ the energy difference in favour of the faceted NPs becomes almost constant with a value close to $1.1 \mathrm{eV}$ per $\mathrm{TiO}_{2}$ unit. Note that this implies that, in terms of total energy, the difference between spherical relaxed and faceted structures grows linearly with the number of $\mathrm{TiO}_{2}$ units explaining the dominance of faceted NP at larger sizes. The large difference in the stability between these NP types can be rationalized by noting the relatively higher proportion of low coordinated atoms in the spherical cut NPs which leads to a small degree of partial amorphization at the surface of the NP.

Experimentally, thermal annealing ${ }^{42,48}$ is often used to reduce defects and to improve the crystalline nature and morphology of titania NPs. ${ }^{49,50}$ Depending on the annealing temperature different structural changes can be observed. ${ }^{51}$ For instance, in absence of annealing, $\mathrm{TiO}_{2}$ thin films tend to exhibit the rutile phase, while annealing at $723 \mathrm{~K}$ causes the anatase phase to become dominant. ${ }^{42}$ Mirroring such experiments, we annealed our spherical cut relaxed NPs using molecular dynamics (MD) 
simulations. Specifically, both faceted and spherical $\mathrm{TiO}_{2} \mathrm{NPs}$ were heated to $1000 \mathrm{~K}$ for 30 ps (full details of the MD simulations are described in Computational Methods Section) followed by a total energy relaxation of the most stable NP structures found during the MD run. For faceted NPs, even for small sizes, we found that annealing does not significantly change the internal structure or morphology. Correspondingly, the energetic stabilisation induced by annealing for such NPs is very small (e.g. $0.02 \mathrm{eV}$ per $\mathrm{TiO}_{2}$ unit for the $\left(\mathrm{TiO}_{2}\right)_{97}$ faceted NP). These results confirm the relative high stability of these NPs. For spherical NPs directly relaxed from bulk cuts, however, we found that annealing substantially increases their energetic stability. Comparing the red and green lines in Fig. 2 we can see that the energetic stabilization induced via annealing is greatest for the smallest spherical NPs but is less significant for the larger NPs. For instance, for $n=50$, the difference in energy between relaxed bulk cut and annealed spherical $\mathrm{TiO}_{2} \mathrm{NPs}$ is almost $0.20 \mathrm{eV} / \mathrm{TiO}_{2}$ in favor of the annealed nanoparticles, whereas for $n=500$ this difference decreases to $0.02 \mathrm{eV} / \mathrm{TiO}_{2}$. The decrease in the extent of stabilization upon annealing as the NP size increases is related both to the MD procedure as well as the potential energy surface (PES) of the NP. For the largest NP sizes considered, both sets of spherical NPs have a similar well-developed crystalline core. As the annealing mainly affects the surface atoms, which become a smaller proportion of the total atoms as the NP size increases, the difference in stability between the nonannealed and annealed NPs is relatively diminished. For smaller NP sizes, the crystalline core contributes proportionally less to the energy, and surface reconstruction becomes a major stabilizing term. For such sizes, the spherical cut NPs can become fully amorphized simply through the structural relaxation optimization process. Analysis of the annealed spherical $\left(\mathrm{TiO}_{2}\right)_{n}$ NP structures shows that the original crystalline bulk core disappears for $n \leq 136$ (i.e. for $\varnothing \leq 2.0 \mathrm{~nm}$ ) in line with other work. ${ }^{22,23}$ For NP sizes with $\varnothing<2 \mathrm{~nm}$ our fits (see Fig. 2) suggest that these amorphized annealed spherical NPs are more energetically stable than faceted anatase NPs. We note that our extrapolated fits in Fig. 2 also indicate that non-crystalline NPs from global optimisation are the most stable NPs in this size regime. Strictly speaking, globally optimised NPs are, by definition, the lowest in energy for any size and thus should include crystalline faceted NPs for large sizes. Following the approach in ref [23], however, these globally optimised NPs are better interpreted as forming the set of lowest energy non-crystalline NPs. The larger members of this set (i.e. $\left(\mathrm{TiO}_{2}\right)_{n}$ for $\left.n=28,35,38\right)$ are very similar to the smallest annealed spherical NPs (i.e. $\left(\mathrm{TiO}_{2}\right)_{n}$ for $\left.n=43,115,136\right)$ with respect to both morphology (i.e. quasi-spherical) and structure (i.e. non-crystalline). As such, we suggest that all six of these NPs should be considered as being a part of a single family of quasi-spherical non-crystalline NPs (see Fig. 1).

To better understand the effect of the annealing process, we analysed the change of the atomic coordination environments, and the coordination number $(\mathrm{CN})$ of Ti and $\mathrm{O}$ atoms in the spherical NPs before and after annealing process. In particular, we focused on the percentage of Ti atoms with 6-fold coordination $\left(\mathrm{Ti}_{6 \mathrm{c}}\right)$ and $\mathrm{O}$ atoms with 3-fold coordination $\left(\mathrm{O}_{3 \mathrm{c}}\right)$, both corresponding to the atomic environments found in the bulk anatase phase. With or without annealing, a clear tendency of an 
increasing percentage $\mathrm{Ti}_{6 \mathrm{c}}$ and $\mathrm{O}_{3 \mathrm{c}}$ with increasing NP size is observed in both set of spherical NPs (Table 1). However, annealed spherical $\mathrm{TiO}_{z} \mathrm{NPs}$ show a higher proportion of $\mathrm{Ti}_{6 e}$ and $\mathrm{O}_{3 \mathrm{e}}$ eentres compared to eorrespondingly sized non-annealed relaxed spherical $\mathrm{TiO}_{z} \mathrm{NPs}_{\mathrm{s}}$ in all cases. This tendency clearly shows that the $\mathrm{Ti}_{6 \mathrm{c}}$ and $\mathrm{O}_{3 \mathrm{c}}$ coordination environments spherical NPs become closer to those in the anatase bulk phase in larger NPs. More specifically, in Table 1 we can see that the percentages of $\mathrm{Ti}_{6 \mathrm{c}}$ and $\mathrm{O}_{3 \mathrm{c}}$ centers in the large $\left(\mathrm{TiO}_{2}\right)_{595}$ annealed spherical NP are very comparable to those in the large faceted crystalline $\left(\mathrm{TiO}_{2}\right)_{455} \mathrm{NP}$, indicating a general increase in the proportion of bulk-like local coordination environments with increasing NP size. This is also justified by the similar $\mathrm{CN}$ values of $\mathrm{Ti}$ and $\mathrm{O}$ atoms corresponding to $\left(\mathrm{TiO}_{2}\right)_{455}$ and $\left(\mathrm{TiO}_{2}\right)_{595}$ NPs as compiled in Table 1. Our analysis matches nicely with previous studies ${ }^{22}$ that shown amorphous $\mathrm{TiO}_{2} \mathrm{NPs}$ composed of $\sim 2 \mathrm{~nm}$ with a highly distorted shell and a strained anatase-like core with a $\mathrm{CN}(\mathrm{Ti})$ of 5.3.

The above described results are schematically shown in the tentative structure versus stability diagram in Fig. 3. Here zero energy corresponds to the lowest energy structure at each size governed by global optimisation and the uppermost black curve follows the metastability of the relaxed spherical cut NPs. The shading in the figure indicates the relative annealing temperature and labels and dashed lines approximately delimit regions where different NP types are more stable. The smallest spherical cut NPs become amorphous upon optimization/annealing and thus non-crystalline NPs dominate for such sizes. With increasing size relaxed spherical cut NPs can persist but are metastable with respect to NPs with thicker amorphous shells and/or fully amorphous NPs. Above a diameter of about $2.7 \mathrm{~nm}$ moderate annealing of spherical relaxed cut NPs will still lead to core-shell type NPs but the most stable annealed NPs are crystalline faceted NPs.

Although, annealed spherical NPs are thus always predicted to be metastable species, depending on experimental conditions, a range of NPs with energies above that of the ideal global minima will typically be produced. For the larger NPs considered $(\varnothing>2 \mathrm{~nm})$, our results show that even after annealing, an anatase crystal core can be maintained in the spherical NPs. Here, although faceted NPs are most energetically stable, experimental NP preparation conditions will largely influence the final morphology and structure. As small anhydrous spherical NPs are often observed, ${ }^{37,52}$ solely thermodynamic stability does not appear to be the main driving force in experiment. Moreover, there is experimental evidence that annealing in vacuum environments appears to be an effective method to activate $\mathrm{TiO}_{2}$ NPs and enhance their visible-light photocatalytic activity. ${ }^{53}$ It thus may be expected that our anhydrous annealed spherical NP may be good models of the spherical NPs in such experiments.

The effect of the shape, size and internal structure of $\mathrm{TiO}_{2} \mathrm{NPs}$ can have a significant influence on the optical absorption energy gap $\left(O_{\text {gap }}\right),{ }^{38}$ which, in turn, dictates their usefulness in photocatalysis. In Fig. 4 we report $O_{\text {gap }}$ values as predicted from the Kohn-Sham orbital energies from our DFT calculations for all considered NPs. Since the PBE functional ${ }^{45}$ exhibits a systematic error with respect to calculated

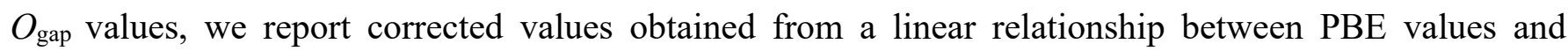


values obtained using the hybrid PBEx (12.5\% Fock exchange) functional. ${ }^{54}$ We note that the PBEx functional reproduces the experimental band gap of anatase and rutile and properly describe the reduced systems. ${ }^{55,56}$ In order to assess the validity of our NP models with respect to experiment, we include a gray shaded region in Fig. 4 delimiting the range of measured $O_{\text {gap }}$ values $(2.85-3.0 \mathrm{eV})$ for dried and annealed anatase $\mathrm{TiO}_{2}$ NPs possessing near spherical morphologies and diameters between $2-10 \mathrm{~nm}^{52}$ For NPs with $\varnothing<2 \mathrm{~nm}$ (i.e. outside of the experimentally measured range) both annealed spherical NPs and faceted NPs have very similar $O_{\text {gap }}$ values in the range 3.2-3.9 eV. Conversely, non-annealed spherical cut NPs in this size range have much lower $O_{\text {gap }}$ values in the range $1.85-2.7 \mathrm{eV}$. We note that all spherical NPs in this small size range are essentially amorphous. Although both annealing and global optimisation are very effective at producing NP structures with few undercoordinated defects, the relaxation of spherical anatase cuts for such sizes leads to very distorted defective structures with gap states and thus small effective energy gaps. In the $\varnothing>2 \mathrm{~nm}$ size regime, although the $O_{\text {gap }}$ values of the faceted NPs remain quite high with respect to experiment, the $O_{\text {gap }}$ values for both sets of spherical NP become significantly lower and much closer to the measured values. The agreement with experiment is particularly good for the annealed spherical NPs which, for all five NP sizes considered, the calculated

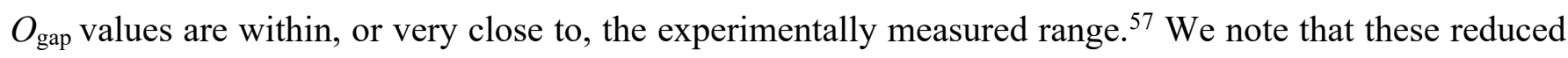
energy gaps are not simply due to the morphology of the spherical NPs. For example, the slightly hydroxylated spherical cut NP models reported by Fazio et al. ${ }^{32}$ maintain energy gaps above that of the anatase bulk crystal. As noted above, such higher-than-bulk energy gaps in small anatase NPs could be rationalised due to quantum confinement. ${ }^{21}$ Although, our spherical NPs are affected by quantum confinement, it appears that the surface structural disorder outweighs this effect to bring the gap below that of anatase. An analysis of the density of states (DOS) near the gap edges of one of larger spherical NPs in our study (see Fig. 4) reveals a broadening of the valence band edge, which reduces their energy gap by $0.6-0.7 \mathrm{eV}$ with respect to that of a similar sized faceted NP.

For all NP families considered, as expected, the optical gap tends to decrease with increasing NP size as the effect of quantum confinement diminishes. We also note that for the largest spherical NPs considered, although the annealed NP has an $O_{\text {gap }}$ value more consistent with experiment, the $O_{\text {gap }}$ value for the relaxed spherical cut NP is significantly lower. This difference is likely due to the fact that annealing increases the energetic stability of the spherical cut NPs by reducing the strain in the near surface structure, and thus reducing the high energy tail of the broadened valence band edge. These results strongly suggest that small experimentally prepared 2-3 nm diameter spherical $\mathrm{TiO}_{2} \mathrm{NPs}$ are likely to have an anatase core and an amorphized shell. ${ }^{22}$ This result is also in line with a previous structural study using classical forcefields. ${ }^{22}$ Our accurate quantum mechanical DFT based calculations allow us to further demonstrate that, relative to faceted crystalline NPs, annealed spherical NPs are metastable core-shell species which display relatively smaller $O_{\text {gap }}$ values through the formation of valence band edge tails and thus should exhibit improved photocatalytic activity. 
A more detailed analysis of the valence band edge in the core shell NPs is shown in Figure 5 where the corresponding partial DOS of the thermally annealed spherical $\left(\mathrm{TiO}_{2}\right)_{257} \mathrm{NP}$ shows that $\mathrm{O}_{2 \mathrm{c}}$ and $\mathrm{Ti}_{4 \mathrm{c}}$ contribute significantly to the broadening. These low coordinated atoms are located mainly in the shell of nanoparticle as depicted in Figure 5b. Note that the presence of a single $\mathrm{O}_{1 \mathrm{c}}$ centre depicted in white colour in Fig. 5b, also contributes to the broadening as shown by dashed line in Fig. 5a. This gap edge broadening, and consequent gap reduction, is analogous to that observed in larger core-shell black $\mathrm{TiO}_{2}$ NPs, and is associated with the disordered structure in their near surface shell. ${ }^{13}$ The metastable black $\mathrm{TiO}_{2}$ NPs are produced by engineering surface disorder in thermodynamically favoured faceted anatase NP by various physico-chemical procedures. Although these methods typically produce NPs with non-stoichiometric shells, ${ }^{13,58,59}$ our results show that significant energy gap engineering can be induced, in principle, by inducing disorder in stoichiometric NPs. Although, terminal defective oxygen species can influence the gap edge, annealing is often found to remove such energetically unfavourable and reactive species in other NPs, leaving only $\mathrm{Ti}_{4 c}$ and $\mathrm{O}_{2 c}$ as the only gap narrowing species. We note that our analysis is also in line with detailed experimental studies of a nanostructured stoichiometric $\operatorname{TiO}_{2}(001)$ surface, where it is suggested that surface $\mathrm{Ti}_{4 \mathrm{c}}$ atoms cause significant band gap narrowing. ${ }^{60}$ As such, these surface science studies and our NP-based results strongly suggest that promoting the formation of surface $\mathrm{Ti}_{4 \mathrm{c}}$ sites could be a general means to significantly elevate photocatalytic activity in stoichiometric $\mathrm{TiO}_{2}$ nanosystems.

Generally, our results highlight that a detailed understanding of the structure, size and morphology of NPs are is crucial in order to understand experimentally measured properties of these important nanosystems. By showing that stoichiometric $\mathrm{TiO}_{2}$ NPs with sizes $<5 \mathrm{~nm}$ have highly tuneable optical energy gaps (above and below that of bulk $\mathrm{TiO}_{2}$ ) our results point to ways in which these factors could be tailored in experiment to optimise the photoactivity of $\mathrm{TiO}_{2} \mathrm{NPs}$.

\section{CONCLUSIONS}

In this work, we have systematically studied the stability of realistic, stoichiometric $\left(\mathrm{TiO}_{2}\right)_{n} \mathrm{NPs}$ containing up to almost 1800 atoms, covering the corresponding NP diameter range from 1.0-3.4 nm. We focus mainly on faceted and spherical morphologies, both with initial structures cut from the bulk anatase crystal structure. After relaxing their geometry, we compare the relative stability and electronic structure properties using accurate all-electron, DFT based calculations. Moreover, we also take into account the effect of the thermal annealing on the structures and properties of these NPs. Our results show that annealing spherical NPs leads to a significant increase in their energetic stability. This effect can be rationalised by the concomitant increase in the proportion of bulk like coordinated atoms upon annealing. For the faceted NPs, however, annealing induced only very minor structural change which causes negligible energetic stabilisation. Non-annealed spherical NPs are always metastable with respect to both 
annealed spherical NPs and faceted NPs. Although faceted NPs are the most stable species for the larger NP sizes considered, for the smaller $\left(\mathrm{TiO}_{2}\right)_{n}$ NPs annealing produces amorphized NP structures. Our analysis of the electronic structure of each NP family reveals that morphology, structure and size, and all have a notable influence on the optical absorption energy gap. Specifically, our calculations confirm that that metastable annealed spherical NPs with anatase cores and amorphized shells and $\varnothing>2 \mathrm{~nm}$ sizes exhibit $O_{\text {gap }}$ values which particularly well match those measured for annealed NPs with 2-10 nm

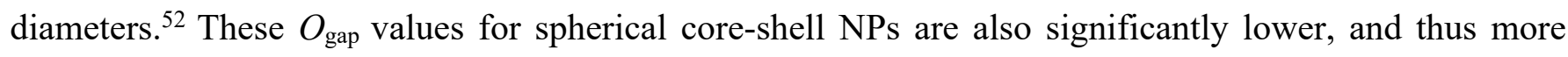
favourable for photocatalysis, than those calculated for the thermodynamically favoured faceted NPs which are above that of bulk anatase. We confirm that these results can be rationalised through a disorderinduced broadening of the valence band edge as observed in experiment for larger black $\mathrm{TiO}_{2} \mathrm{NPs}_{\text {. Our }}$ study using pure $\mathrm{TiO}_{2}$ NPs also implies it is not necessary to employ procedures which introduce nonstoichiometry or dopant atoms for this band gap reducing effect. Instead the presence of $\mathrm{Ti}_{4 \mathrm{c}}$ and $\mathrm{O}_{2 \mathrm{c}}$ sites in the disordered shell of the core-shell NPs are found to be sufficient to produce valence band tails to reduce the energy gap. The impressive agreement between our calculations and experiment for core-shell NPs with 2-3 nm diameters implies that the conditions employed to prepare these NPs tends to lead to kinetically trapped core-shell structures. Our results clearly show that stoichiometric $\mathrm{TiO}_{2} \mathrm{NPs}_{\mathrm{s}}$ with sizes $<5 \mathrm{~nm}$ have highly tuneable optical energy gaps (above and below that of bulk $\mathrm{TiO}_{2}$ ) and point to ways in which these factors could be tailored in experiment to optimise their photoactivity.

\section{COMPUTATIONAL METHODS}

The stability of faceted and spherical stoichiometric $\left(\mathrm{TiO}_{2}\right)_{n}$ NPs with structure derived from anatase bulk phase (Fig. 1) has been studied for $n$ running from 29 to 595. The faceted NPs were obtained via Wulff constructions, ${ }^{61}$ which relates the shape of the NP to the areas and relative stabilities of the exposed surfaces. The bipyramidal NPs exhibit the most stable (101) surface and display octahedral symmetry (Fig. 1a), whereas the truncated octahedral NPs display both (101) and (001) surfaces (Fig. 1b). ${ }^{38}$ Spherical $\mathrm{TiO}_{2}$ NPs (Fig. 1c) were generated by cutting spheres of the desired diameters from an the anatase crystal structure following a procedure reported in other works, ${ }^{62-64}$ while ensuring that the $\mathrm{TiO}_{2}$ stoichiometry was always preserved. For all NPs, the initial structure was relaxed using total energy minimization as described below. In addition to relaxation, molecular dynamics (MD) simulations runs were carried out to mimic an annealing process aimed to induce structural reorganization of the surface while keeping the internal structure of the particle as intact as possible. The MD simulations were performed within the canonical ensemble (NVT) and consisted of 5 ps of equilibration time followed by $30 \mathrm{ps}$ of production at $1000 \mathrm{~K}$ using an interatomic potential specifically parameterised for nanoscale $\mathrm{TiO}_{2}$ in a previous work. ${ }^{23}$ Structures and energies were sampled every $0.01 \mathrm{ps}$ and the lowest five energies obtained during the MD run were selected and further relaxed by using DFT based methods as described below. We note that this annealing process maintains the core of the particle with the anatase 
structure while the surface is amorphized for $\left(\mathrm{TiO}_{2}\right)_{n}$ NPs with $n>163$, whereas for smaller NPs the anatase core was unstable to amorphization.

The structures of the smallest global optimized NPs were taken from ref [23]. For the larger NPs, the initial structures cut from bulk anatase were fully optimised employing DFT based calculations carried out without symmetry constraints employing the PBE functional. ${ }^{45}$ Optical gaps were estimated from calculations using the PBEx hybrid functional ${ }^{55}$ or, for the largest ones, extrapolated following a recently reported relationship. ${ }^{54}$ The DFT based calculations explicitly include all electrons and the electron density is described through a numerical atom-centered orbital basis set, as implemented in FHIaims package. ${ }^{46}$ A light grid and tier-1 basis set was used, which has an accuracy comparable to a TZVP Gaussian-type orbital basis set. ${ }^{38}$ The convergence threshold for atomic forces during the relaxation of the structure of the NPs is set to $10^{-3} \mathrm{eV} \AA^{-1}$. The zero-order regular approximation (ZORA) is employed to include relativistic effects. ${ }^{65,66}$

\section{CONFLICTS OF INTEREST}

There are no conflicts to declare.

\section{ACKNOWLEDGEMENTS}

This research was supported by the Spanish MINECO/FEDER CTQ2015-64618-R and Spanish Structures of Excellence María de Maeztu program through grant MDM-2017-0767 and, in part, by Generalitat de Catalunya (grants 2017SGR13 and XRQTC). A. M.-G. thanks to Spanish MEC for the Juan de la Cierva postdoctoral grant (FJCI-2015-23760) and F. I. acknowledges additional support from the 2015 ICREA Academia Award for Excellence in University Research. Computational time at the MareNostrum supercomputer has been provided by the Barcelona Supercomputing Centre (BSC) through the grant of the Partnership for Advanced Computing in Europe (PRACE) under the EXCIPHOCAT project (2016163940). 
Table 1. Pencentages of $\mathrm{Ti}_{6 \mathrm{c}}$ and $\mathrm{O}_{3 c}$ centres (with respect to the total number of $\mathrm{Ti}$ and $\mathrm{O}$ centres respectively) in optimized $\left(\mathrm{TiO}_{2}\right)_{115},\left(\mathrm{TiO}_{2}\right)_{237}$, and $\left(\mathrm{TiO}_{2}\right)_{595}$ spherical NPs before and after the annealing process. The faceted $\left(\mathrm{TiO}_{2}\right)_{455} \mathrm{NP}$ is also included for comparison. Note that the coordination numbers of centres are obtained using a cutoff radius for a Ti-O of $2.4 \AA$.

\begin{tabular}{|c|c|c|c|c|c|c|c|c|}
\hline & \multicolumn{6}{|c|}{ Spherical NPs } & \multirow{2}{*}{\multicolumn{2}{|c|}{$\begin{array}{c}\text { Faceted NP } \\
\left(\mathrm{TiO}_{2}\right)_{455}\end{array}$}} \\
\hline & \multicolumn{2}{|c|}{$\left(\mathrm{TiO}_{2}\right)_{115}$} & \multicolumn{2}{|c|}{$\left(\mathrm{TiO}_{2}\right)_{237}$} & \multicolumn{2}{|c|}{$\left(\mathrm{TiO}_{2}\right)_{595}$} & & \\
\hline & $\mathbf{T i}_{6 c}$ & $\mathbf{O}_{3 \mathrm{c}}$ & $\mathbf{T i}_{6 \mathrm{c}}$ & $\mathrm{O}_{3 \mathrm{c}}$ & Ti $_{6 c}$ & $\mathbf{O}_{3 \mathrm{c}}$ & $\mathbf{T i}_{6 \mathrm{c}}$ & $\mathbf{O}_{3 \mathrm{c}}$ \\
\hline Before annealing & 5.2 & 19.6 & 48.9 & 59.4 & 61.2 & 68.8 & 58.2 & 77.8 \\
\hline After annealing & 9.6 & 25.6 & 43.5 & 53.2 & 59.1 & 66.9 & 58.2 & 77.8 \\
\hline & \multicolumn{8}{|c|}{ Coordination Number $(\mathrm{CN})$ of Ti atom } \\
\hline Before annealing & 4.4 & & & 3 & & 5.5 & & 5.5 \\
\hline \multirow[t]{2}{*}{ After annealing } & 4.6 & & & 2 & & 5.4 & & 5.5 \\
\hline & \multicolumn{8}{|c|}{ Coordination Number (CN) of $\mathrm{O}$ atom } \\
\hline Before annealing & 2.2 & & & .7 & & 2.8 & & 2.8 \\
\hline After annealing & 2.3 & & & .6 & & 2.7 & & 2.8 \\
\hline
\end{tabular}


Fig. 1 Structures of $\left(\mathrm{TiO}_{2}\right)_{n}$ NPs in the size range $n=29-595$ with: (a) bipyramidal, (b) cuboctahedral, (c) spherical morphologies, and (d) selected globally optimised structures from ref. [23]. Further details about the bipyramidal and cuboctahedral $\mathrm{TiO}_{2}$ nanoparticles can be found in ref. [38]. Red and blue spheres correspond to $\mathrm{O}$ and $\mathrm{Ti}$ atoms, respectively. The dash-dotted line delimits a set of amorphous $\left(\mathrm{TiO}_{2}\right)_{n}$ NPs. 

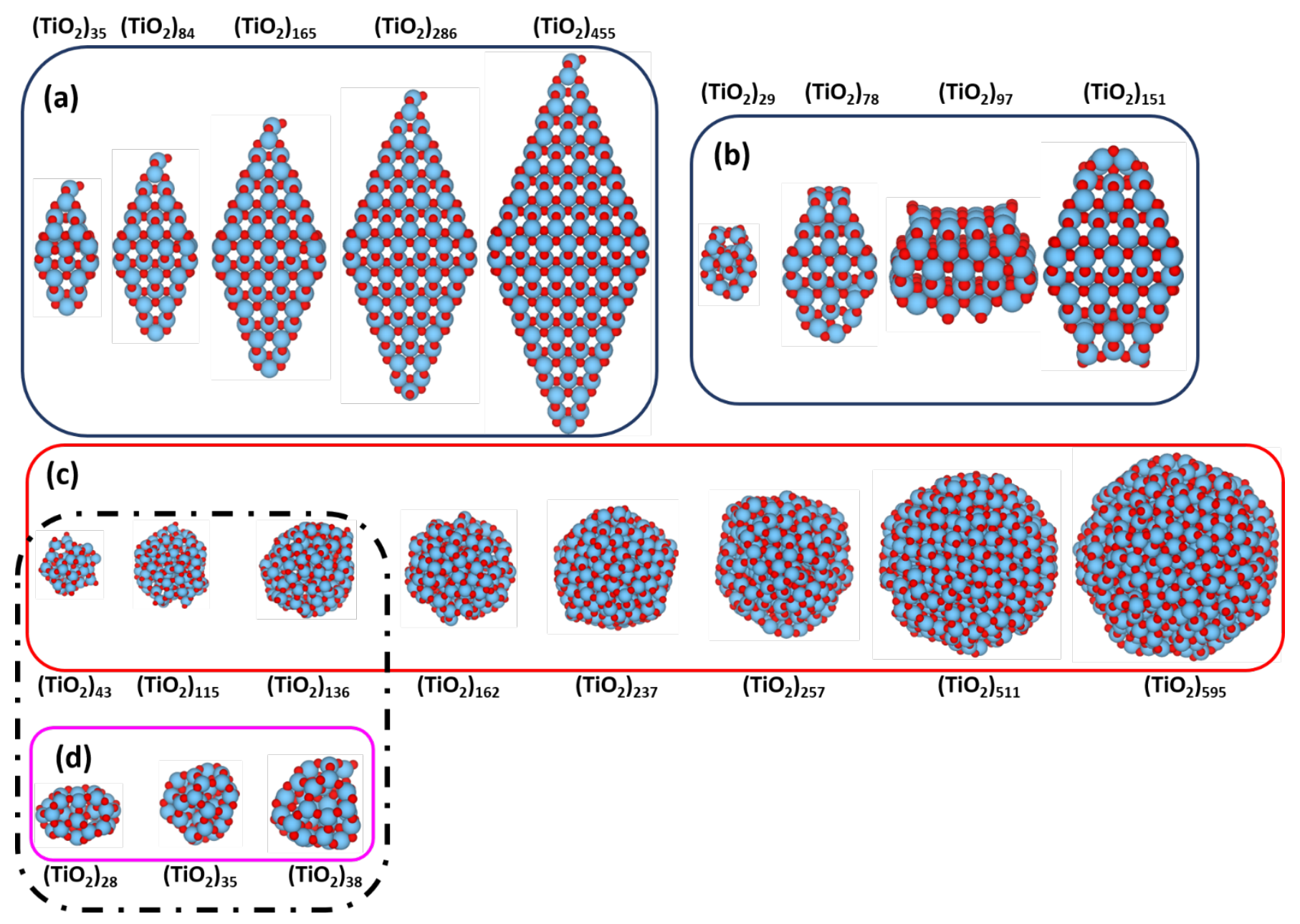
Fig. 2 Evolution of the energy per $\mathrm{TiO}_{2}$ unit relative to the anatase bulk phase (set to zero) of: faceted (blue), spherical (red), annealed spherical (green), and global optimized (pink) $\mathrm{TiO}_{2} \mathrm{NPs}$ as a function of the number of $\mathrm{TiO}_{2}$ units $(n)$. An approximate diameter is included on the upper $x$-axis. The fits for each set are depicted by solid lines (fitting parameters are reported in the ESI). Note that faceted NPs correspond to the bipyramidal and cuboctahedral geometries depicted in Fig. 1a and 1b, respectively. Spherical NPs are shown in Figure 1c. Further information regarding the globally optimized $\mathrm{TiO}_{2} \mathrm{NPs}$ (Fig. 1d) can be found in Ref. [23].

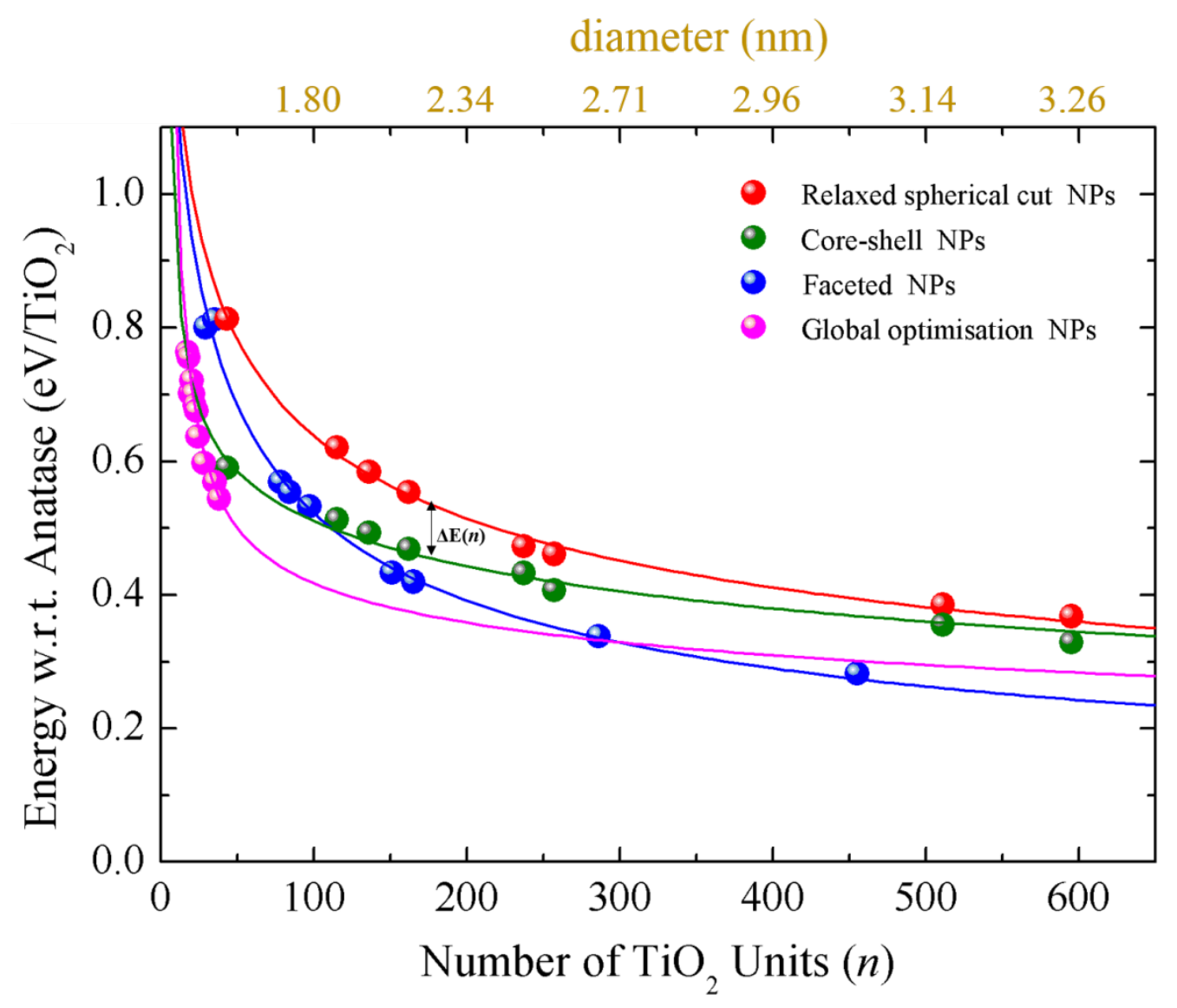


Fig. 3 Tentative structure versus stability diagram for $\mathrm{TiO}_{2} \mathrm{NPs}$ with respect to the relaxed spherical cut NPs (upper black line) based on the fitted tendencies and the NP structures corresponding to the data points in Fig. 2.

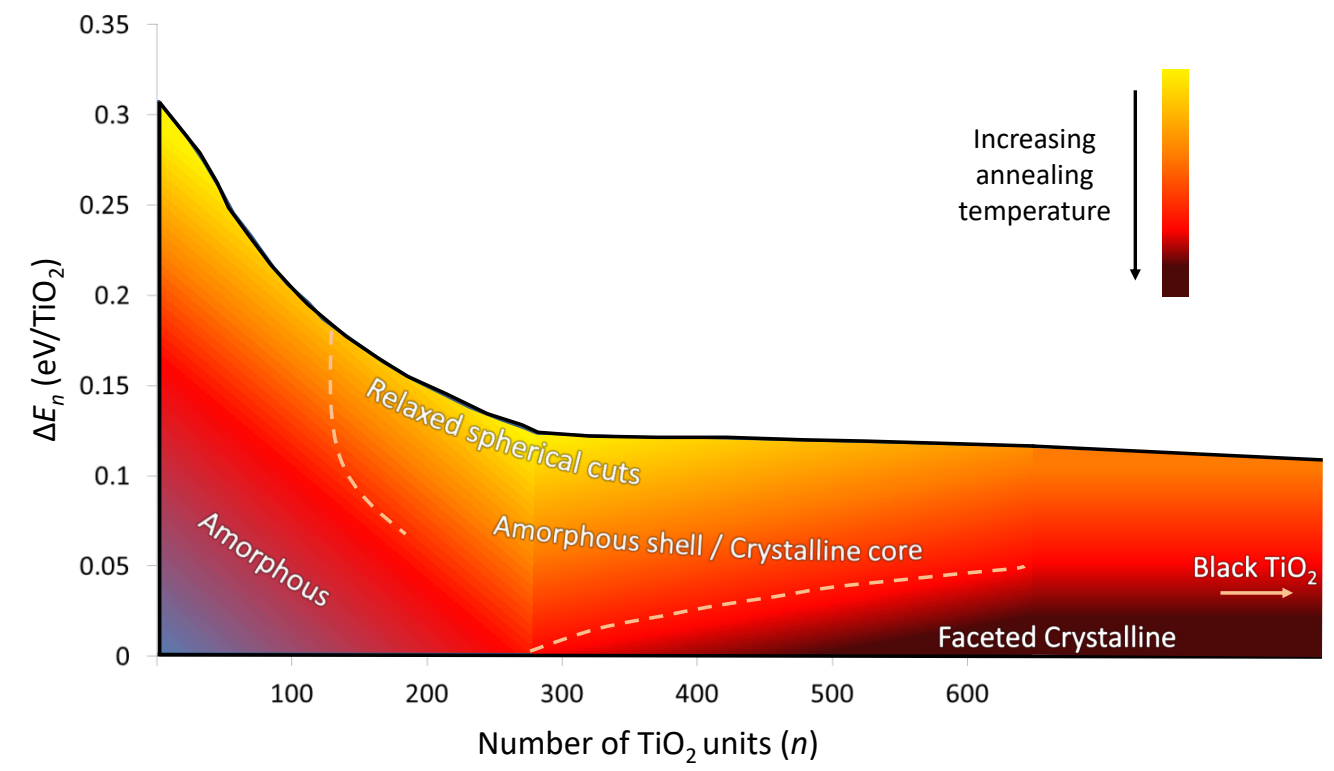


Fig. 4 (a) The optical gap $\left(O_{\text {gap }}\right)$ as estimated via the difference between the highest occupied molecular orbital (HOMO) and the lowest unoccupied molecular orbital (LUMO) of faceted, spherical (with and without annealing), and global optimised $\mathrm{TiO}_{2}$ NPs calculated using the hybrid PBEx exchange correlation functional. ${ }^{55}$ Note that the data for the relaxed spherical cuts and the annealed spherical coreshell NPs were determined following the correlation found between $O_{\text {gap }}(\mathrm{PBE})$ and $O_{\text {gap }}(\mathrm{PBEx})$ recently reported in Ref. 54. The grey horizontal bar represents experimentally measured $O_{\text {gap }}$ results for $\mathrm{TiO}_{2} \mathrm{NPs}$ with near spherical shapes and with diameters ranging from 2 to $10 \mathrm{~nm} .^{52}$ The dashed line indicates the band gap in bulk $\mathrm{TiO}_{2}$ anatase phase (i.e. $\left.3.2 \mathrm{eV}\right)$. (b) The density of state (DOS) of faceted $\left(\mathrm{TiO}_{2}\right)_{455}$ and direct cut and annealed spherical $\left(\mathrm{TiO}_{2}\right)_{511} \mathrm{NPs}$ - corresponding to the circled data points in (a). The structural disorder near the surfaces of the spherical NPs creates broadened tails in their valence band (VB) edges, leading to the reduction of the energy gap. Conversely the conduction band (CB) is similar for all three considered NPs.

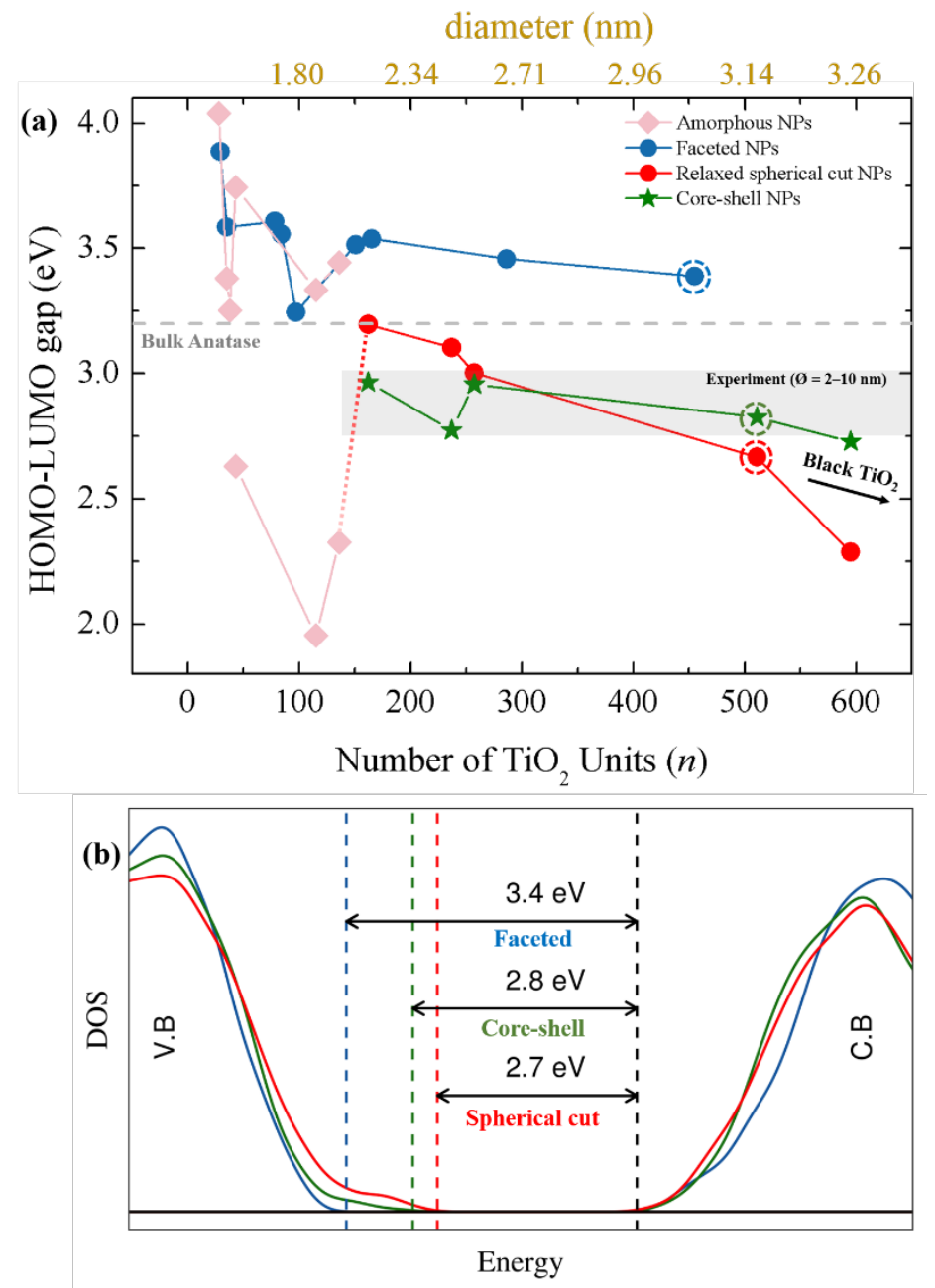


Fig. 5 (a) Partial DOS plot of an annealed core-shell $\left(\mathrm{TiO}_{2}\right)_{257} \mathrm{NP}$ with energies given eV. Each colour indicates the contribution of $\mathrm{Ti}$ or $\mathrm{O}$ atoms with different coordinations (the dashed line shows the contribution of a single terminating $\mathrm{O}_{1 \mathrm{c}}$ centre). (b) Cross sectional cut through the annealed core-shell $\left(\mathrm{TiO}_{2}\right)_{257} \mathrm{NP}$ where $\mathrm{Ti}$ and $\mathrm{O}$ atoms with different coordination are shown by the same colour coding in the partial DOS plot. The cut clearly shows a bulk-like crystalline core (dominated by $\mathrm{Ti}_{6 c}$ and $\mathrm{O}_{3 \mathrm{c}}$ centres) and a disordered shell (dominated by $\mathrm{Ti}_{4 c}, \mathrm{Ti}_{5 \mathrm{c}}$ and $\mathrm{O}_{2 \mathrm{c}}$ centres). Note that the white sphere corresponds to the single $\mathrm{O}_{1 \mathrm{c}}$ centre.

(a)

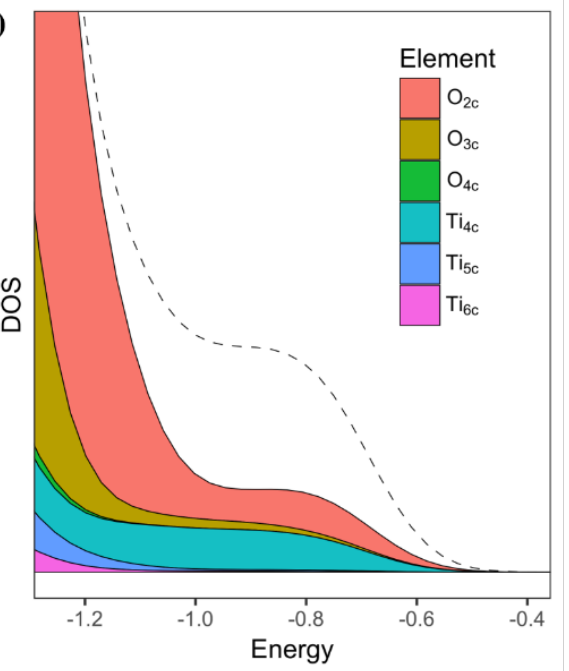

(b)

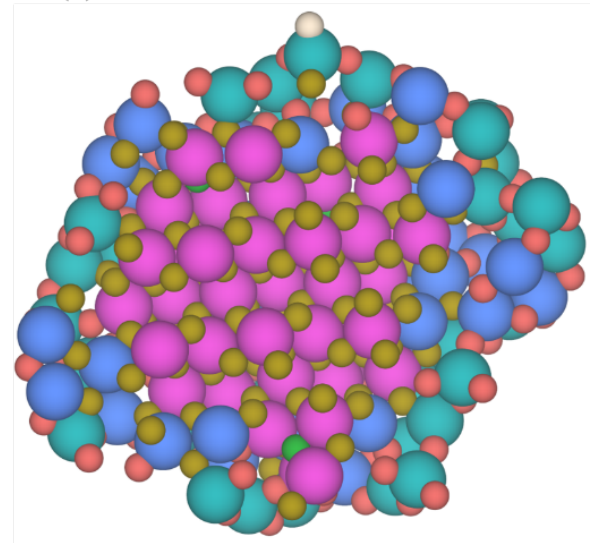




\section{References}

(1) L. Liu and X. Chen, Chem. Rev., 2014, 114, 9890-9918.

(2) H. Xu, S. Ouyang, L. Liu, P. Reunchen, N. Umezawa and J. Ye, J. Mater. Chem. A, 2014, 2, 12642-12661.

(3) A. Fujishima and K. Honda, Nature, 1972, 238, 37-38.

(4) X. Chen and S. S. Mao, Chem. Rev., 2007, 107, 2891-2959.

(5) M. G. Walter, E. L. Warren, J. R. McKone, S. W. Boettcher, Q. Mi, E. A. Santori and N. S. Lewis, Chem. Rev., 2010, 110, 6446-6473.

(6) M. Murdoch, G. I. Waterhouse, M. A. Nadeem, J. B. Metson, M. A. Keane, R. F. Howe, J. Llorca and H. Idriss, Nat. Chem., 2011, 3, 489-492.

(7) H. Reiche and A. J. Bard, J. Am. Chem. Soc., 1979, 101, 3127-3128.

(8) D. A. Tryk, A. Fujishima and K. Honda, Electrochim. Acta, 2000, 45, 2363-2367.

(9) A. L. Linsebigler, G. Q. Lu and J. T. Yates, Chem. Rev., 1995, 95, 735-758.

(10) D. O. Scalon, C. W. Dunnill, J. Buckeridge, S. A. Shevlin, A. J. Logsdail, S. M. Woodley, C. R. A. Catlow, M. J. Powell, R. G. Palgrave, I. P. Parkin, G. W. Watson, T. W. Keal, P. Sherwood, A. Walsh and A. Sokol, Nat. Mater., 2013, 12, 798-801.

(11) M. Anpo, and M. J. Takeuchi, J. Catal., 2003, 216, 505-516.

(12) S. G. Kumar, and L. G. Devi, J. Phys. Chem. A, 2011, 115, 13211-13241.

(13) L. Liu, P. Y. Yu, X. Chen, S. S. Mao, D. Z. Shen, Phys. Rev. Lett., 2013, 111, 065505 (1-5).

(14) I. Nakamura, N. Negishi, S. Kutsuna, T. Ihara, S. Sugihara and K. Takeuchi, J. Mol. Catal. A: Chem., 2000, 161, 205-212.

(15) X. Pan, M.-Q. Yang, X. Fu, N. Zhang and Y.-J. Xu, Nanoscale, 2013, 5, 3601-3614.

(16) A. S. Barnard and P. Zapol, Phys. Rev. B, 2004, 70, 235403 (1-12).

(17) X. Chen, C. Li, M. Graetzel, R. Kostecki and S. S. Mao, Chem. Soc. Rev., 2012, 41, 7909-7937.

(18) D. Selli, G. Fazio and C. Di Valentin, Catalysts, 2017, 7, 357 (1-24).

(19) Y.-F., Li and Z.-P. Liu, J. Am. Chem. Soc., 2011, 133, 15743-15752.

(20) X. Chen, L. Liu, P. Y. Yu, and S. S. Mao, Science, 2011, 331, 746-750.

(21) K. C. Ko, S. T. Bromley, J. Y. Lee and F. Illas, J. Phys. Chem. Lett., 2017, 8, 5593-5598.

(22) H. Zhang, B. Chen, J. F. Banfield and G. A. Waychunas, Phys. Rev. B, 2008, 78, 214106-214101.

(23) O. Lamiel-García, A. Cuko, M. Calatayud, F. Illas and S. T. Bromley, Nanoscale, 2017, 9, 10491058.

(24) R. Darkins, M. L. Sushko, J. Liu, and D. M. Duffy, Phys. Chem. Chem. Phys., 2014, 16, 94419447.

(25) R. Menzel, A. M. Peiró, J. R. Durrant and M. S. P. Shaffer, Chem. Mater., 2006, 18, 6059-6068.

(26) D. V. Bavykin, J. M. Friedrich and F. C. Walsh, Adv. Mater., 2006, 18, 2807-2824.

(27) M. Cargnello, T. R. Gordon and C. B. Murray, Chem. Rev., 2014, 114, 9319-9345. 
(28) C.-T. Dinh, T.-D. Nguyen, F. Kleitz and T.-O. Do, ACS Nano, 2009, 3, 3737-3743.

(29) C. Chen, R. Hu, K. Mai, Z. Ren, H. Wang, G. Qian and Z. Wang, Z. Cryst. Growth Des., 2011, 11, 5221-5226.

(30) T. R. Gordon, M. Cargnello, T. Paik, F. Mangolini, R. T. Weber, P. Fornasiero and C. B. Murray, J. Am. Chem. Soc., 2012, 134, 6751-6791.

(31) G. Fazio, D. Selli, L. Ferraro, G. Seifert and C. Di Valentin, ACS Appl. Mater. Interfaces., 2018, 10, 29943-29953.

(32) G. Fazio, L. Ferrighi, and C. Di Valentin, J. Phys. Chem. C, 2015, 119, 20735-20746.

(33) K. Shirai, G. Fazio, T. Sugimoto, D. Selli, L. Ferraro, K. Watanabe, M. Haruta, B. Ohtani, C. Di Valentin, and Y. Matsumoto, J. Am. Chem. Soc., 2018, 140, 1415-1422.

(34) G. Fazio, L. Ferrighi and C. Di Valentin, Nano Energy, 2016, 27, 673-689.

(35) X. Chen, I. Liu, M. A. Marcus, W.-C. Wang, N. A. Oyler, M. E. Grass, B. Mao, P.-A- Glans, P. Y. Yu, J. Guo, and S. S. Mao, Sci. Rep., 2013, 3, 1510 (1-7).

(36) S. Mugundan, B. Rajamannan, G. Viruthagiri, N. Shanmugam, R. Gobi and P. Praveen, Appl. Nanosci., 2014, 5, 449-456.

(37) A. Ansari, S. Sachar and S. S. Garje, New J. Chem., 2018, 42, 13358-13366.

(38) O. Lamiel-García, K. C. Ko, J. Y. Lee, S. T. Bromley and F. Illas, J. Chem. Theory Comput., 2017, 13, 1785-1793.

(39) O. V. Prezhdo, W. R. Duncan and V. V. Prezhdo, Prog. Surf. Sci., 2009, 84, 30-68.

(40) F. Nunzi, L. Storchi, M. Manca, R. Giannuzzi, G. Gigli and F. De Angelis, ACS Appl. Mater. Interfaces, 2014, 6, 2471-2478.

(41) S. Sugapriya, R. Sriram and S. Lakshmi, Optik, 2013, 124, 4971-4975.

(42) A. Dussan, A. Bohórquez and H. P. Quiroz, Appl. Surf. Sci., 2017, 424, 111-114.

(43) X. Zhou, E. Wierzbicka, N. Liu and P. Schmuki, Chem. Commun., 2019, 55, 533-536

(44) F. Viñes, O. Lamiel-García, F. Illas and S. T. Bromley, Nanoscale, 2017, 9, 10067-10074.

(45) J. P. Perdew, K. Burke and M. Ernzerhof, Phys. Rev. Lett., 1996, 77, 3865-3868.

(46) V. Blum, R. Gehrke, P. Hanke, P. Havu, V. Havu, X. Ren, K. Reuter and M. Scheffler, Comput. Phys. Commun., 2009, 180, 2175-2196

(47) R. Johnson, Masters Series in Physics and Astronomy in Atomic and Molecular Clusters, Taylor and Francis, 2002.

(48) S. Liu, J. Yu and M. Jaroniec, Chem. Mater., 2011, 23, 4085-4093.

(49) D. P. Padiyan and S. T. Nishanthi, Trans. Mat. Res. Soc. Japan, 2013, 38, 127-130.

(50) D. Selli, G. Fazio and C. Di Valentin, J. Chem. Phys., 2017, 147, 164701 (1-12).

(51) G. Okeke, R. B. Hammond and J. Antony, Chem. Eng. Sci., 2016, 146, 144-158. 
(52) V. M. Ramakrishnan, M. Natarajan, A. Santhanam, V. Asokan and D. Velauthapillai, Mater. Res. Bull., 2018, 97, 351-360.

(53) M. Samiee and J. Luo, Mater. Lett., 2013, 98, 205-208.

(54) Á. Morales-García, R. Valero and F. Illas, Phys. Chem. Chem. Phys., 2018, 20, 18907-18911.

(55) K. C. Ko, O. Lamiel-García, J. Y. Lee and F. Illas, Phys. Chem. Chem. Phys., 2016, 18, $12357-$ 12367.

(56) Á. Morales-García, R. Valero and F. Illas, J. Chem. Theory Comput., 2017, 13, 3746-3753.

(57) A. Naldoni, M. Allieta, S. Santangelo, M. Marelli, F. Fabbri, S. Cappelli, C. L. Bianchi, R. Psaro and V. Dal Santo, J. Am. Chem. Soc., 2012, 134, 7600-7603.

(58) S. G. Ullattil, S. B. Narendranath, S. C. Pillai and P. Periyat, Chem. Eng. J., 2018, 343, 708-736

(59) S. Chen, Y. Wang, J. Li, Z. Hu, H. Zhao, W. Xie, and Z. Wie, Mater. Res. Bull., 2018, 98, 280287.

(60) H. Ariga, T. Taniike, H. Morikawa, M. Tada, B. K. Min, K. Watanabe, Y. Matsumoto, S. Ikeda, K. Saiki and Y. Iwasawa, J. Am. Chem. Soc., 2009, 131, 14670-14672.

(61) G. Wulff, Kristallogr., 1901, 34, 449-530.

(62) E. Rabani, J. Chem. Phys., 2001, 115, 1493-1496.

(63) H. Zhang, F. Huang, B. Gilbert and J. F. Banfield, J. Phys. Chem. B, 2003, 107, 13051-13060.

(64) H. Zhang, B. Gilbert, F. Huang and J. F. Banfield, Nature, 2003, 424, 1025-1029.

(65) C. Chang, M. Pelissier and M. Durand, Phys. Scr., 1986, 34, 394-404.

(66) E. van Lenthe, R. van Leeuwen, E. J. Baerends and J. G. Snijders, Int. J. Quantum Chem., 1996, 57, 281-293. 
TOC

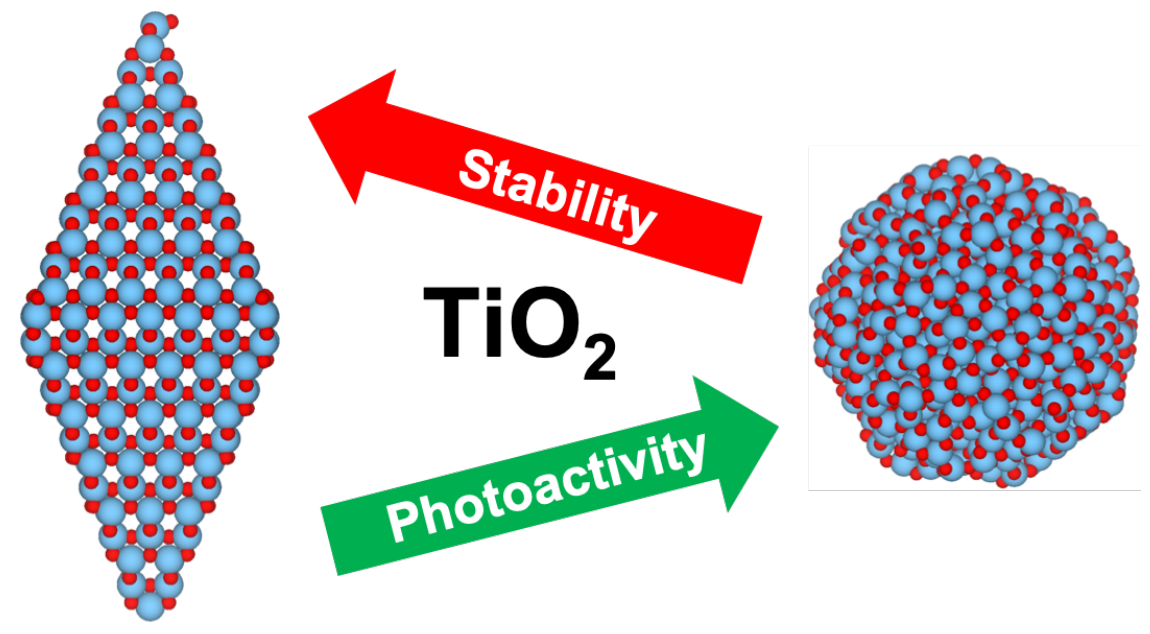

